\title{
Parallel Line Grouping Based on Interval Graphs
}

\author{
Peter Veelaert \\ Hogent \\ Schoonmeersstraat 52, 9000 Ghent, Belgium \\ Peter.Veelaert@hogent. be
}

\begin{abstract}
We use an interval graph to model the uncertainty of line slopes in a digital image. We propose two different algorithms that group lines into classes of lines that are parallel, or almost parallel. This grouping is strongly based on the Helly-type property of parallelism in the digital plane: a group of lines is digitally parallel if and only if each pair of lines is digitally parallel. As a result, the extraction of parallel groups is reduced to the extraction of cliques in the interval graph generated by the slope intervals. Likewise, the extraction of lines that are almost parallel becomes equivalent to the detection of subgraphs that resemble cliques.
\end{abstract}

\section{Introduction}

In this paper we consider the graph-theoretical properties of parallel line grouping in a digital image, mostly from the computational viewpoint. This work is part of an ongoing effort to define a sound mathematical basis for the extraction of geometric structure from an image while taking into account the uncertainty that is present. In previous work we already considered some basic geometric properties such as collinearity, parallelism and concurrency [8], and we studied some of the algorithmic aspects of the theory [9]. In this paper we propose algorithms that classify lines into groups of parallel lines, taking into account the uncertainty of edge positions and the fact that parallelism is not an equivalence relation in the digital plane.

Any algorithm that extracts geometric structure must take into account the uncertainty on the position of the pixels. This uncertainty has several causes: the digitization process, the localization errors introduced by the edge detector, and the possible errors introduced by the line extraction algorithms, e.g., RANSAC or the Hough Transform. In fact, uncertainty can even be an artificial parameter, e.g., to detect automatically in an image vanishing points of the perspective projection, a first step is to extract groups of lines that "almost" parallel.

In the approach proposed here, the uncertainty of point positions is modeled by defining for each point a small uncertainty region in which the point can lie, without knowing its exact position. This may be compared to the more general approach as advocated by Durrant-Whyte and others where one defines for each position a probability distribution [1]. These distributions are then used to derive new geometric distributions, e.g., for the parameters of a line passing 
through two points. By contrast, instead of computing precise distributions, we only calculate in which region a distribution is non-zero. The advantage of this simplification is that we can go much further in the extraction of geometric information, without computing complicated probability measures. By comparison, also Lowe makes simplifying assumptions regarding the distribution of lines in an image and introduces an empirical significance measure for parallelism [5]. Lowe's interesting view is inspired by concepts from perceptual organization, but it remains empirical, without a strict formal introduction of uncertainty.

In euclidian geometry grouping into parallel classes would be trivial, as euclidian parallelism is an equivalence relation. In a digital image uncertainty on positions causes uncertainty on the slopes of lines, and parallelism is therefore no longer an equivalence relation. We must find partitions that "resemble" equivalence classes. In this work we explain how this problem can be reformulated as finding groups of lines that resemble cliques in the so-called graph of parallel pairs. This reformulation is possible because parallelism in the digital plane turns out to be a Helly-type property: a collection of lines is digitally parallel if each its pairs of lines is digitally parallel [8]. That is, the Helly-type property holds for the entire collection as soon as it holds for each of the collection's $n$-membered subcollections, where $n=2$.

In this paper we show how a classification into parallel groups depends on two factors: first, a measure for the uncertainty on pixel positions, which we call the acceptable thickness, and second, an optimization criterion, which determines how much parallelism is required in a group of parallel lines. Thus, we may require that each group consists of lines that are all parallel to each other, or we may impose the weaker requirement that it suffices that each group contains at least one line to which all other lines in the group are parallel.

In Section 2 we give a brief overview of the concepts of digital straightness, collinearity, parallelism, and concurrency as they are used in this work. Section 3 introduces the so-called graph of parallel pairs, which represents all parallel relations between line segments. In Section 4 two different algorithms are proposed that use the graph of parallel pairs to extract large groups of parallel line segments. We give some concluding remarks in Section 5.

\section{Brief Overview of Geometric Properties}

In this section we briefly review results obtained in previous work [8,9]. Since image pixels lie on a rectangular grid, we assume that all pixels are part of the digital plane, although this is not strictly necessary for what follows. The digital plane $\mathbb{Z}^{2}$ consists of points that have integral coordinates. A digital set $S$ is a subset of the digital plane.

Several issues have influenced the development of mathematical discretization schemes. For computer generated images discretization schemes are used to obtain a digital representation of a euclidian object. For natural images they provide a simplified model of the complicated discretization process of digital image formation, i.e., a model for a CCD camera. Finally, for natural as well as 
computer images, the discretization scheme can be used to examine the inverse process, i.e., given a digitized image How can we reconstruct the original image and the geometric structure contained within it?

In this paper we are mostly interested in the last issue. During discretization the precise knowledge about position of geometric objects is lost. Or in other words, given the digital image we are uncertain about the position of objects in the original image. We shall model this uncertainty by an uncertainty region that we associate with each grid point. The discretization process that coincides naturally with this notion of uncertainty is the discretization by dilation scheme developed by Heijmans and Toet [2]. Let $U$ denote a set in $\mathbb{R}^{2}$, let $A$ be a second subset of $\mathbb{R}^{2}$, called the structuring element, and let $A_{p}$ be the translate of $A$ by $p$. Then the discretization by dilation of $U$ consists of all points $p \in Z^{2}$ for which $A_{p} \cap U$ is non-empty [2,7].

Furthermore, to simplify the exposition, in this paper we shall restrict ourselves to one particular form of discretization by dilation, that is, we use a simple variant of grid-intersect discretization. To model the uncertainty of its position, for each digital point $p=(x, y)$, we introduce as translate of the structuring element the vertical line segment $C_{p}(\tau)$, which comprises all points $(x, b) \in \mathbf{R}^{2}$ that satisfy $y-\tau / 2 \leq b<y+\tau / 2$. Here $\tau$ is a positive real number, called the acceptable thickness. Up to a certain multiplication factor, this notion of acceptable thickness coincides with Réveillès's notion of arithmetical thickness of a discrete straight line [6]. Fig. 1 shows several digital sets, their vertical segments, and straight lines that transverse these segments. To simplify what follows even further, we shall only discuss the extraction of straight lines of the form $y=\alpha x+\beta$, where the slope $\alpha$ satisfies $-1<\alpha<1$. We assume that each set $S$ contains at least two points with distinct $x$-coordinates.

Although the foregoing restrictions do not change our results in a fundamental way, they allow us to discard several special cases discussed more thoroughly in previous work [8], where we show, for example, that for lines whose slope lies between -1 and 1 , vertical segments are sufficient to model position uncertainty. Also in previous work, other cells than vertical line segments have been considered in a more general framework where we examine the modeling of uncertainty in the multitude of parameter spaces that arise during the hierarchical grouping of line segments [10].

Definition 1. A digital set $S$ is called digitally straight if all segments $C_{p}(\tau)$, $p \in S$ can be transversed by a common straight line.

Note that the above definition of straightness is more general than the standard definition of a digitally straight line, where one chooses $\tau=1$, and where additional connectivity constraints must be satisfied.

Definition 2. Let $S_{1}, \ldots, S_{n}$ a finite collection of finite digitally straight sets, and let $A_{i}$ denote a euclidian straight line. We define the following digital geometric properties, illustrated in Fig. 1:

- The sets $S_{i}$ are called digitally collinear if there exist $n$ collinear line segments $A_{1}, \ldots, A_{n}$ where each $A_{i}$ transverses all the segments of $S_{i}$; 
- The sets in $S_{i}$ are called digitally parallel if there exist $n$ parallel straight lines $A_{1}, \ldots, A_{n}$ where each $A_{i}$ transverses all the segments of $S_{i}$;

- The $S_{i}$ are called digitally concurrent if there exist $n$ straight lines $A_{1}, \ldots, A_{n}$ that meet in a common point and where each $A_{i}$ transverses all the segments of $S_{i}$.
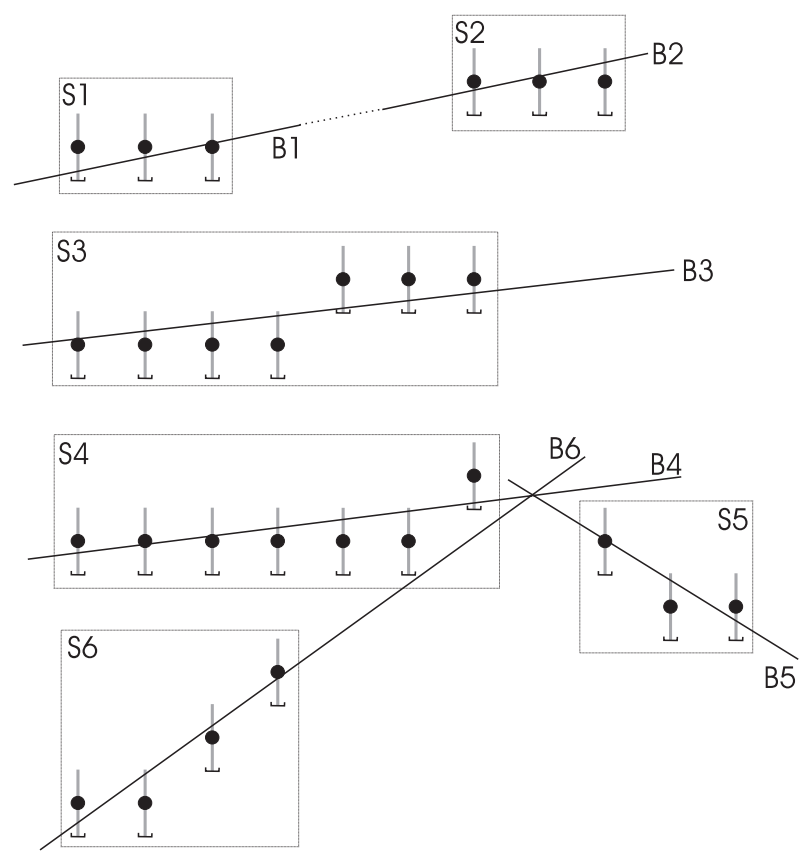

Fig. 1. $S_{1}$ and $S_{2}$ are digitally collinear; $S_{3}$ and $S_{4}$ are digitally parallel; $S_{4}, S_{5}$ and $S_{6}$ are digitally concurrent

The following result is crucial [8]:

Theorem 1. Let $\mathcal{S}=\left\{S_{1}, \ldots, S_{n}\right\}$ a finite collection of finite digitally straight sets. Then the collection $\mathcal{S}$ is digitally parallel if each subcollection containing two sets of $\mathcal{S}$ is digitally parallel.

According to Theorem 1 all knowledge about parallelism can be derived from the parallelism of pairs. Although we focus in this paper mainly on the graphtheoretical algorithms, we give a brief overview of the geometric algorithms that are needed to determine whether a pair of lines is digitally parallel [9].

Definition 3. Let $S$ be a finite digital set that contains at least two points with distinct $x$-coordinates, and let $\tau$ be a chosen acceptable thickness with $0<\tau$. 
Then the domain of $S$, denoted as $\operatorname{dom}_{x}(S ; \tau)$, is the set of all parameter points $(\alpha, \beta) \in \mathbb{R}^{2}$ that satisfy the following system of inequalities:

$$
-\tau / 2<\alpha x_{i}+\beta_{i}-y_{i} \leq \tau / 2, \quad\left(x_{i}, y_{i}\right) \in S .
$$

In other words, the domain contains the parameters of all the euclidian lines that transverse the vertical segments of $S$. In general, the domain of a large and sufficiently elongated set is small, since the parameters of the lines transversing all these segments can vary only within a small range.

Consider for example the collection of digital sets representing lines in Fig. 2, whose domains are shown in Fig. 3, for an acceptable thickness $\tau=2$. The long line segment $O$ has a small domain, while the short segment $K$ has a large domain. In fact, since each point of a set corresponds to two halfplanes in the parameter space, the domain of a large set is the intersection of a large number of halfplanes, and, in general, the domain will therefore be small. Furthermore, a domain of a digital set with $N$ points is a convex bounded set, and can be computed in $O(N \log N)$ time as an intersection of $2 N$ halfplanes [9]. When we let $\tau$ vary, a domain gets larger for increasing values of $\tau$. If the acceptable thickness is too small, however, the domain of a set will be empty. For given set $S$, let $D \subset \mathbb{R}$ be the set of acceptable thicknesses for which the domain of $S$ is non-empty. The thickness of $S$ is defined as the infimum of $D$ [8].

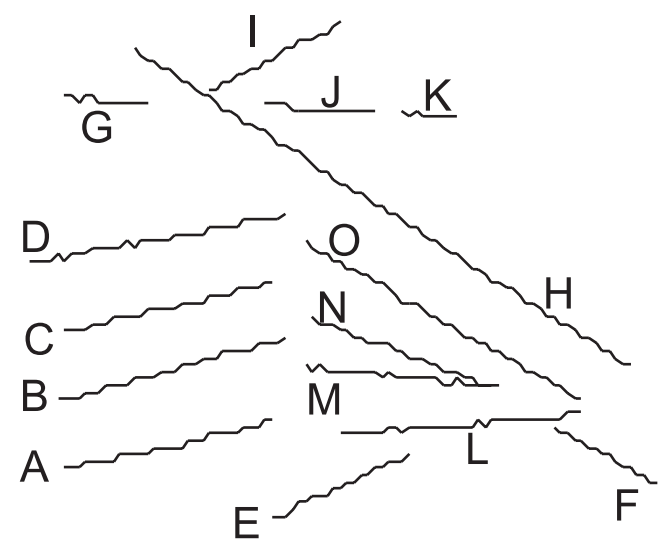

Fig. 2. A collection of lines

To reformulate the digital geometric concepts of Definition 2 in terms of domains, we need one more notion, i.e., the projection of a domain upon the $\alpha$-axis in the $(\alpha, \beta)$ parameter plane. For a subset $S$ of $\mathbb{R}^{2}$, let $\pi_{\alpha}\left(\operatorname{dom}_{x}(S ; \tau)\right)$ denote the interval that results by projecting the domain of $S$ upon the $\alpha$-axis. The interval $\pi_{\alpha}\left(\operatorname{dom}_{x}(S ; \tau)\right)$ has the following geometrical meaning: $\pi_{\alpha}\left(\operatorname{dom}_{x}(S ; \tau)\right)$ is equal to the open interval ] $\alpha^{1}, \alpha^{2}$ [, where $\alpha^{1}$ is the infimum of the slopes of the 


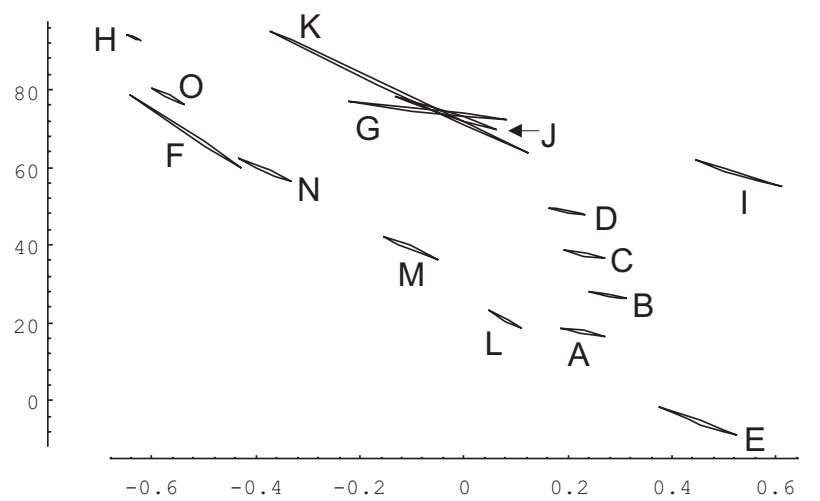

Fig. 3. Domains of lines for the acceptable thickness $\tau=2$

euclidian lines that transverse the vertical segment associated with the points in $S$, and where $\alpha^{2}$ is the supremum. Fig. 4(a) shows the slope intervals that result by projecting the domains in Fig. 3 upon the $\alpha$-axis. It follows almost immediately that two lines are digitally parallel if and only if their slope intervals have a non-empty intersection $[8,9]$.

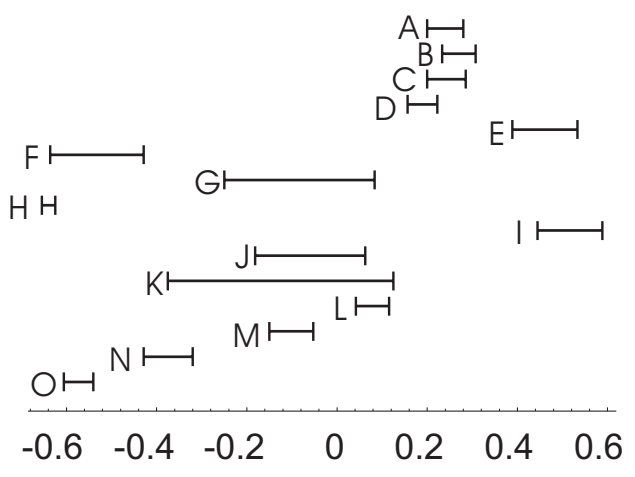

(a)

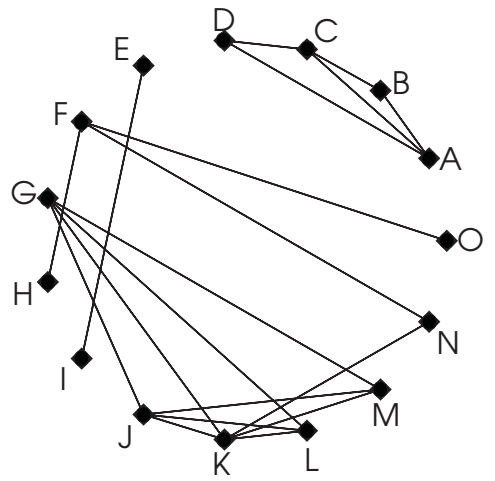

(b)

Fig. 4. (a) The intervals that result from the projection of the domains, and (b) the corresponding graph of parallel pairs 


\section{Graph of Parallel Pairs}

We shall represent parallelism by the graph shown in Fig. 4(b), called the graph of parallel pairs. In this graph two vertices are connected by an edge if the corresponding lines are digitally parallel. Theorem 1 remains essential here, since it allows us to conclude that any complete subgraph (or clique) is digitally parallel, i.e. the cliques $G J K L$ and $G J K M$ in Fig. 4(b), . In other words, this conclusion is valid because parallelism in the digital plane is a Helly type property, that is, we can draw a conclusion for the entire set by examining all pairs, i.e, the edges of the graph.

Before proceeding we must briefly review the definitions and properties of graphs that we need (see, e.g., [11]). Let $G$ be a simple graph, i.e, a graph that contains no loops or double edges. An independent set is a set of vertices that does not contain any pair of adjacent vertices. A dominating set is a set of vertices such that each vertex in $G$ is adjacent to at least one vertex in the dominating set. A vertex cover is a set of vertices such that each edge in $G$ is incident with at least one vertex in the vertex cover. A coloring assigns an index to each vertex so that two adjacent vertices always receive distinct indices. An optimal coloring uses a minimum number of indices, i.e., the chromatic number of the graph. A graph is perfect if every induced subgraph has an independent set meeting all its maximum cliques, that is, cliques of maximal size.

In the graph of parallel pairs, two vertices are joined by an edge when the slope intervals of the two lines intersect. Therefore, this graph falls into the category of interval graphs. Similarly, the complement of an interval graph belongs to the category of comparability graphs. Interval graphs as well as comparability graphs are perfect [11], which has important consequences from the computational viewpoint. In general, finding an optimal coloring, a minimum dominating set, a minimum vertex covering, minimum clique covering, or maximum dependent set are NP-hard problems for graphs in general $[3,11]$. As soon as we have a perfect graph, however, each of these sets and coverings can found in polynomial time [3]. Interval graphs possess even stronger properties, and the above sturctures can often be found in linear time.

\section{Classification of Lines into Parallel Groups}

In euclidian geometry a graph of parallel pairs would consist of disjoint cliques. Since parallelism in the digital plane is not an equivalence relation we must look at techniques that extract subgraphs that are similar to cliques, and in this way approximate the equivalence relation of euclidian geometry. Fig. 5 shows an application, where long lines with slope between -1 and 1 have been extracted from the image of a building. Fig. 5(b) shows the graph of parallel pairs for $\tau=2$. In this particular application we wanted to detect automatically some of the vanishing points in the image. The idea is to look for three or more lines that meet at a common point and whose slopes are almost equal. To reduce the number of possible combinations, one of the essential steps is to extract good candidate groups of lines that are almost parallel. 


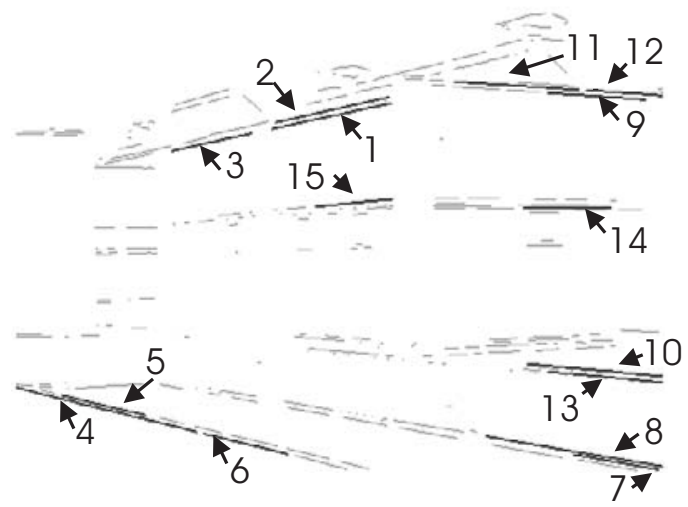

(a)

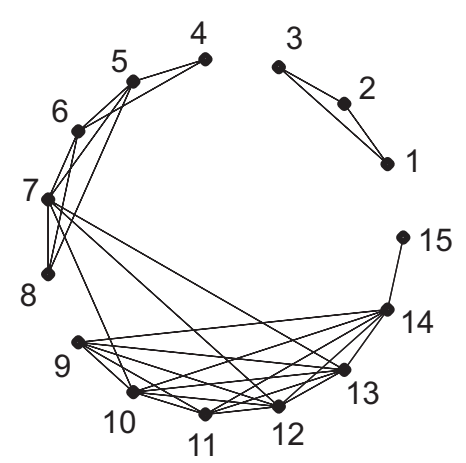

(b)

Fig. 5. (a) Lines detected in image of building with horizontal Sobel operator. (b)Graph of parallel pairs of longest lines for acceptable thickness $\tau=2$

We shall propose two algorithms that partition a graph of parallel pairs into subgraphs that resemble cliques. Clearly, the partitioning will depend on the amount of parallelism that is required within each group. The first algorithm looks for a minimal number of groups in which parallelism is maximal, that is, each line in the group must be parallel to each other group member. By contrast, we could look for groups with a minimal amount of parallelism, by extracting the connected components of the graph. The second algorithm, imposes a more natural requirement, it looks for groups in which there is at least one member to which all other lines are parallel.

\subsection{Classification Based on Minimum Clique Coverings}

Covering the graph of parallel pairs by a minimum number of cliques is equivalent to finding an optimal coloring of its complement which is a comparability graph. The algorithm that we propose here is a modification of an existing coloring algorithm that uses the partial ordering induced by a comparability graph (see [11], page 201). We can use the fact, however, that the comparability graph considered here is the complement of an interval graph, and that the partial ordering can be derived directly from the intervals. Each line $S$ yields an interval $\left.\pi_{\alpha}\left(\operatorname{dom}_{x}(S ; \tau)\right)=\right] a_{i}, b_{i}[$ of slopes. Let $] a_{1}, b_{1}[, \ldots,] a_{n}, b_{n}$ [ the intervals be sorted according to their lowest boundary $a_{i}$ and assume that we have reassigned indices such that $a_{i} \leq a_{j}$ whenever $i<j$. Note that the ordering of this sequence is not unique. Furthermore, the intervals induce an ordering on the complementary graph. To be precise, we let $S_{i}<S_{j}$ whenever the slope intervals do not intersect and we have $b_{i}<a_{j}$. 
Algorithm 1. Partitioning into parallel subsets (Equivalent to optimal coloring of complement of interval graph). We start by selecting from the sorted sequence the interval $\left.B_{1}=\right] a_{j}, b_{j}$ [ for which $b_{j}$ takes a minimum value. To this interval and to all the intervals that intersect it we assign the first color, and we remove these colored intervals from the sequence. We then proceed iteratively by assigning the $i$ th color to the interval $\left.B_{i}=\right] a_{k}, b_{k}$ [ for which $b_{k}$ takes the minimum value in the remaining sequence and to all the intervals that intersect it. The algorithm yields a partitioning into a minimum number of cliques.

In addition, after coloring the vertices, if we select one vertex of each color, then it is clear that we obtain a maximal independent set, that is, an independent set that cannot be extended further. That this independent set is also the largest possible follows from the Perfect Graph Theorem, according to which the maximal size of an independent set of a perfect graph is equal to the minimum number of colors needed to color its complement.

Correctness of the Algorithm. The algorithm produces a valid coloring for the complement of the interval graph. In fact, we claim that the intervals are only assigned the same color when their common intersection is non-empty. After assigning $m$ colors, let ] $a_{k}, b_{k}$ [ be an interval for which $b_{k}$ takes a minimum value in the remaining sequence. Then the real number $b_{k}$ must lie in all those intervals in the remaining sequence that intersect $] a_{k}, b_{k}\left[\right.$, since otherwise $b_{k}$ would not be a minimum. Thus, all intervals that are in the same coloring class have a common non-empty intersection. As a result they are all mutually incomparable, and therefore they form an independent set in the comparability graph. Furthermore, the coloring must be optimal, since if the algorithm uses $m$ colors then there are at least $m$ mutually disjoint intervals, or in other words, there is a clique of size $m$ in the comparability graph.

Example. Fig. 4(a) shows the slope intervals that correspond to the lines in Fig. 2. If we restrict ourselves to the lines of the largest component of Fig. 4(b), we find the following partitioning: $\{H, F\},\{O\},\{N, K\},\{M, G, J\},\{L\}$. This corresponds to a coloring of the complement of the graph of parallel pairs. Since we have a minimum number of color classes, the algorithm yields a partitioning into a minimum number of classes of parallel lines, or in other words a minimum clique partitioning of the interval graph. The lines $\{H, O, N, M, L\}$ form a maximum independent set.

\subsection{Classification Based on a Minimum Dominating Set}

A partitioning into a minimal number of parallel sets may not always yield the most natural result. Consider the example in Fig. 6. If we apply Algorithm 1, we find the following partitioning: $\{A, B\},\{C, D, E\},\{F\}$. The symmetry apparent in the graph of parallel pairs and the intervals does not occur in the grouping. In fact, in this example any minimum clique partitioning is asymmetric. We now propose a second technique that requests less parallelism in the groups, and often 
leads to more natural results. It is a covering based on a minimum dominating set of the graph. Recall that a set of vertices is dominating if each vertex is adjacent to at least vertex in the dominating set. We shall group the lines such that each group contains one vertex of a dominating set and all its neighbors. Thus, in each group there will be a line to which all other lines in the group are parallel. Note that distinct groups may not be disjoint.

Let $S$ denote the set of intervals, and assume that the intervals $] a_{1}, b_{1}[, \ldots$, ]$a_{n}, b_{n}$ [ have been assigned indices according to an ordering on their left boundaries, i.e., $a_{i} \leq a_{j}$ whenever $i<j$.

(a)

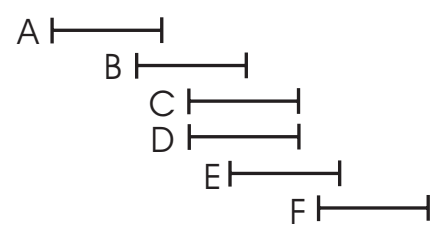

(b)

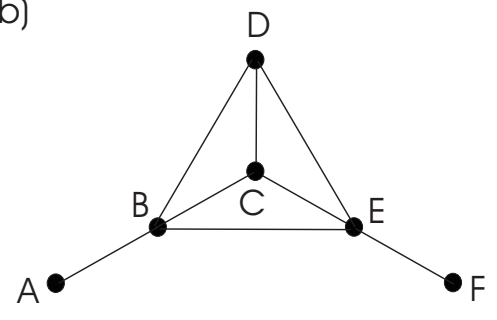

Fig. 6. (a) Slope intervals of a set of lines; (b) The corresponding graph of parallel pairs

Algorithm 2. Covering with parallel subsets (Equivalent to minimum dominating set for interval graphs). We first set the dominating set $D$ equal to the empty set. Let $\left.B_{j}=\right] a_{j}, b_{j}$ [ be the interval in the sorted sequence for which $b_{j}$ takes a minimum value. We look in the sorted sequence for an interval $] a_{i}, b_{i}$ [ that intersects $] a_{j}, b_{j}$ [ and for which $b_{i}$ takes a maximum value. We add $] a_{i}, b_{i}$ [ to the dominating set $D$, and we remove $] a_{i}, b_{i}$ [ and all the intervals that intersect it from the sorted sequence. We proceed iteratively by taking the next interval ]$a_{k}, b_{k}$ [ in the remaining sequence for which $b_{k}$ takes a minimum value.

Correctness of the Algorithm. Clearly, the resulting set $D$ is a dominating set, since it includes for each interval at least one interval that intersects it. Furthermore, the dominating set has minimum size, which can be seen as follows. Let $\left.D=\{] p_{1}, q_{1}[,] p_{2}, q_{2}[, \ldots)\right\}$ be the dominating set found by the algorithm, and let $] a_{j}, b_{j}$ [ be the interval for which $b_{j}$ is a minimum in the starting sequence.

First, for the interval $] a_{j}, b_{j}$ [ there must be at least one interval in any dominating set that intersects it. Let $E^{0}$ be any minimum dominating set, and let ]$a_{k}, b_{k}$ [ be an interval in $E^{0}$ that intersects $] a_{j}, b_{j}[$. Then we claim that the set $\left.E^{1}=\left(E^{0} \backslash\right] a_{k}, b_{k}[) \cup\right] p_{1}, q_{1}[$ is also a minimum dominating set. It suffices to prove that any interval that intersects $] a_{k}, b_{k}[$, also intersects $] p_{1}, q_{1}[$. Let $] a_{m}, b_{m}[$ 
be an interval that intersects $] a_{k}, b_{k}[$. Suppose that $] a_{m}, b_{m}[$ also intersects $] a_{j}, b_{j}[$. Then, $] p_{1}, q_{1}$ [ also intersects $] a_{m}, b_{m}\left[\right.$, since $b_{j}$ is common to all the intervals that intersect $] a_{j}, b_{j}$ [, otherwise $b_{j}$ would not be a minimum value. Suppose $] a_{m}, b_{m}$ [ does not intersect $] a_{j}, b_{j}\left[\right.$, then $b_{j}<a_{m}$. It follows that $] a_{m}, b_{m}$ [ must intersect ]$p_{1}, q_{1}[$, because among the set of all intervals $] a_{i}, b_{i}[$ that intersect $] a_{j}, b_{j}[$, which includes $] a_{k}, b_{k}\left[\right.$, we have $b_{i} \leq q_{1}$.

Next, it is clear that the set $\left.E^{1} \backslash\right] p_{1}, q_{1}$ [ must be a minimum dominating set for the set formed by all intervals in $S$ that do not intersect $] a_{j}, b_{j}[$, because otherwise $E^{1}$ itself would not have minimum size. Thus, by the same reasoning as above, we can prove that there exists a minimum dominating set $E^{2}$ that contains $] p_{2}, q_{2}$ [, and therefore there must a minimum dominating set containing both $] p_{1}, q_{1}$ [ and $] p_{2}, q_{2}[$. By proceeding iteratively, it follows that $D$ is indeed a dominating set of minimal size.

If we apply this algorithm to the intervals of Fig. 4(a), then we find $\{F, K\}$ as a minimum dominating set. This dominating set yields the following grouping: $\{F, O, N, H\}$ and $\{K, L, M, J, G, N\}$. Note that the line $N$ belongs to both groups, as it is parallel to $F$ as well as $K$. Although this grouping is not a partitioning, it leads to a more natural result than the minimum clique partitioning algorithm.

One may expect that the dominating set method generally produces fewer groups than a minimum clique partition. This is indeed the case, as the following properties hold for any graph: (i) An independent set is a dominating set if and only if it is maximal; Thus, the size of minimum dominating set cannot be larger than the size of a maximum independent set; (ii) Any vertex cover is a dominating set; Thus, the size of minimum dominating set cannot be larger than the size of a minimum vertex cover; (iii) The complement of an independent set is a vertex cover. Hence the following relation follows:

\#(minimum dominating set) $\leq$

min (\# (maximum independent set), $|S|-\#$ (maximum independent set)) .

For example, for the complete graph $K_{n}$, the size of a maximum independent set is 1 , and a minimum vertex cover has size $n-1$. For the star shaped graph, defined as the graph with $n$ vertices where there are only edges between one central vertex and each other vertex, the size of the maximum independent set is $n-1$, and the size of a minimum vertex cover is 1 . In both cases, however, the size of a minimum dominating set is 1 .

\section{Concluding Remarks}

In future work, we will examine the application of the proposed algorithms to discretization by dilation schemes that use structuring elements that are more general than the vertical line segments used here. This extension seems to be rather straightforward, the major consequence being that the computation of the domains of the line segments becomes more intricate. The extension to other 
more recent digitization schemes such as the topology preserving scheme proposed by Latecki et al. [4], and the Hausdorff discretization of Ronse and Tajine seems less obvious, however [7]. In the first place both schemes have been developed to have certain desirable properties such as preservation of topology and reconstructability of the original object (when the grid resolution is increased). To capture the notion of position or line slope uncertainty was not the primary goal of these two schemes.

Furthermore, we are also looking how parallel line grouping can be integrated into a larger framework that also involves collinearity, concurrency and proximity of lines. In this regard, we expect that graph theory will remain inportant. In fact, whatever the way uncertainty in a digital image is represented, it is rather natural to represent the uncertainty of line slopes by slope intervals, which has led us to interval graphs. We concluded that algorithms that perform parallel line grouping can do this by extracting groups of vertices that resemble cliques, and we discussed two possibilities: (i) extract the cliques themselves, or (ii) extract neighborhoods of dominating sets. Both techniques have been well examined in graph theory, and we showed how they provide a formal way to extract parallel groups of lines.

\section{References}

1. H. F. Durrant-Whyte: Uncertain Geometry. In Geometric Reasoning, Eds. Kapur and Mundy, 447-481 (1989) 530

2. H. J. A. M. Heijmans and A. Toet: Morphological sampling. Computer Vision, Graphics \& Image Processing: Image Understanding 54, 384-400 (1991) 532

3. M. Grötschel, L. Lovász, and A. Schrijver: Geometric Algorithms and Combinatorial Optimization. New York: Springer-Verlag, 2nd Ed. (1993) 536

4. L. J. Latecki, C. Conrad, and A. Gross: Preserving topology by a digitization process. J. Math. Imaging and Vision. 8, 131-159 (1998) 541

5. D. Lowe: 3-D object recognition from single 2-D images. Artificial Intelligence 31, 355-395 (1987) 531

6. J.-P. Réveillès: Géométrie discrète, calcul en nombres entiers et algorithmique. Thèse de Doctorat d'Etat. Université Louis Pasteur, Strasbourg (1991) 532

7. C. Ronse and M. Tajine: Discretization in Hausdorff space. J. Math. Imaging and Vision. 12, 219-242 (2000) 532, 541

8. P. Veelaert: Geometric constructions in the digital plane. J. Math. Imaging and Vision. 11, 99-118 (1999) 530, 531, 532, 533, 534, 535

9. P. Veelaert: Algorithms that measure parallelism and concurrency of lines in digital images. Proceedings of SPIE's Conference on Vision Geometry VIII, (Denver), SPIE, 69-79 (1999) 530, 531, 533, 534, 535

10. P. Veelaert: Line grouping based on uncertainty modeling of parallelism and collinearity. Proceedings of SPIE's Conference on Vision Geometry IX, (San Diego), SPIE (2000) 532

11. D. B. West: Introduction to Graph Theory. Upper Saddle River: Prentice Hall (1996) 536, 537 